\title{
Systematic review of implementation strategies for risk tables in the prevention of cardiovascular diseases
}

\author{
Ben van Steenkiste \\ Richard Grol \\ Trudy van der Weijden
}

Centre for Quality of Care Research, School for Public Health and Primary Care (Caphri), Maastricht University, Maastricht, The Netherlands
Correspondence: Ben van Steenkiste Department of General Practice, Centre for Quality of Care Research, Maastricht University, PO Box 616,6200 MD Maastricht, The Netherlands

Tel +3I 433882329

Fax +3 I 43 36| 9344

Email ben.vansteenkiste@hag.unimaas.nl
Background: Cardiovascular disease prevention is guided by so-called risk tables for calculating individual's risk numbers. However, they are not widely used in routine practice and it is important to understand the conditions for their use.

Objectives: Systematic review of the literature on professionals' performance regarding cardiovascular risk tables, in order to develop effective implementation strategies.

Selection criteria: Studies were eligible for inclusion if they reported quantitative empirical data on the effect of professional, financial, organizational or regulatory strategies on the implementation of cardiovascular risk tables. Participants were physicians or nurses.

Outcome measure: Primary: professionals' self-reported performance related to actual use of cardiovascular risk tables. Secondary: patients' cardiovascular risk reduction.

Data collection and analysis: An extensive strategy was used to search MEDLINE, EMBASE, CINAHL, and PSYCHINFO from database inception to February 2007.

Main results: The review included 9 studies, covering 3 types of implementation strategies (or combinations). Reported effects were moderate, sometimes conflicting and contradictory. Although no clear relation was observed between a particular type of strategy and success or failure of the implementation, promising strategies for patient selection and risk assessment seem to be teamwork, nurse led-clinics and integrated IT support.

Conclusions: Implementation strategies for cardiovascular risk tables have been sparsely studied. Future research on implementation of cardiovascular risk tables needs better embedding in the systematic and problem-based approaches developed in implementation science.

Keywords: systematic review, implementation, cardiovascular diseases, primary prevention

\section{Introduction}

Reducing levels of modifiable cardiovascular risk factors is a key goal in the prevention of cardiovascular diseases (CVD), and guidelines are an important means of achieving this goal (Graham et al 2006). Primary preventive treatment is targeted at patients who are asymptomatic but are at elevated absolute 10-year CVD risk. Identification of persons at high risk is guided by so-called risk tables, which are tools designed for the assessment of an individual's risk score. Numerous CVD-risk tables are available (Sheridan et al 2003; Will 2005). Risk tables differ in many respects, eg, in the way risks are framed and presented, the number of risk factors included, outcome measures, interpretation, and indications for medical treatment (Thomas et al 1999; Conroy et al 2003; Sheridan et al 2003). These differences, and the validity of the prediction function, are still subjects of study and debate (Brindle et al 2006; Graham et al 2006; Wang et al 2006).

Despite certain weaknesses or restrictions, there is no doubt about the value of risk tables for preventive treatment in everyday patient care. However, extensive efforts to publish and disseminate such tables have not yet resulted in the desired level of 
implementation in routine practice (De Koning et al 2004). A systematic review of the effectiveness of cardiovascular risk tables as regards risk reduction in daily practice was inconclusive in terms of the effect on patients' risk reduction, because application of the risk tables by the professionals was not optimal. It became clear that a serious implementation problem needed to be addressed. The poor uptake of risk tables by the physicians was confirmed in observational studies (Brindle et al 2006). A risk calculator for risk management was only routinely used by $17 \%$ of American family physicians (Eaton et al 2006), and less than half of the physicians in two European studies (De Muylder et al 2004; Graham et al 2006). Moreover, Australian GPs reported that - if they used cardiovascular risk assessment tools at all - they used them in a restricted manner, only as an aid to patient education (Torley et al 2005). Apparently, there is a gap between the high risk approach advocated in the prevention of CVD and the actual use of risk tables in routine practice. A high-risk approach supported by risk tables seems to be an innovation that is not easy to implement. It is important to investigate what implementation strategies are most effective to ensure a good uptake of the risk tables in normal practice.

The literature reports on many different methods, strategies, and measures to introduce innovations, guidelines, best practices, or new procedures into clinical practice (Grimshaw 2003). Effective implementation of innovations seems to be more successful with strategies for implementation that are tailored to the specific goals, target group and setting (Grol 1997, 2001, 2003; Shaw 2005). Therefore, the objective of our study was to systematically review the literature on health professionals' performance with respect to cardiovascular risk tables, in order to search for implementation strategies that enable professionals to use cardiovascular risk tables effectively in the prevention of CVD.

\section{Methods}

\section{Design}

Systematic literature review.

\section{Studies included}

A study was eligible for inclusion in the review if it met the following inclusion criteria:

- quantitative empirical data were reported;

- a professional, financial, organizational, or regulatory strategy was used to implement a CVD-risk table (Table 1);

- participants were physicians or nurses;
- the primary outcome measure was professionals' performance regarding the actual use of cardiovascular risk tables, and the secondary outcome measure was patients' cardiovascular risk reduction by improving one or more modifiable risk factors, eg, blood pressure, cholesterol, smoking, etc.

No restrictions were used as regards setting.

\section{Search strategy}

We elaborated on the extensive search strategy used by Brindle and colleagues (2006), who recently reviewed the accuracy and impact of risk assessment in the primary prevention of CVD. We added free-text search terms related to the implementation of cardiovascular risk tables. Table 2 details the terms used to search MEDLINE. Appropriate adaptations of the search syntax were made when searching EMBASE, CINAHL, and PSYCHINFO. We covered the period from database inception to February 2007. Reference lists of included articles were scanned to identify additional study reports. Languages were restricted to Dutch, English, and German.

\section{Data extraction}

Titles and abstracts of all retrieved references were scanned by one author $(\mathrm{BvS})$, after which two reviewers (BvS and TvdW) independently assessed the remaining set of articles for definitive inclusion or exclusion. The final set of included studies was assessed by one of the authors (BvS) on the basis of the following methodological aspects; study design, type of intervention, participants (profession), setting (location of care and country), and methods (unit of allocation and quality criteria); his findings were checked by another author (TvdW). The final decisions on inclusion and data extraction per study were made by consensus.

\section{Data analysis}

The examination of the methodological quality of the studies was guided by the Data Collection Template (July 2002) of The Cochrane Effective Practice and Organisation of Care (EPOC) group, see www.epoc.uottawa.ca/tools.htm.

\section{Results}

Over 1800 titles and abstracts were scanned, and the full text of 37 articles was assessed. Ten of these articles ( 2 articles reporting on the same study) met our inclusion criteria, resulting in 9 studies being included. The included studies were categorized according to their main type of intervention(s) in EPOC terms. These are summarized in Table 3A-C. 
Table I Types of intervention, listed according to EPOC ${ }^{a}$

\begin{tabular}{|c|c|c|c|}
\hline Professional interventions & Financial interventions & Organizational interventions & Regulatory interventions \\
\hline Distribution of educational materials & Provider interventions & Provider oriented interventions & Changes in medical liability \\
\hline Educational meetings. & Fee-for-service & Revision of professional roles & Management of patient complaints \\
\hline Local consensus processes & Prepaid & Clinical multidisciplinary teams & Peer review \\
\hline Educational outreach visits & Capitation & Formal integration of services & Licensure \\
\hline Local opinion leaders & Provider salaried service & Skill mix changes & \\
\hline Patient-mediated interventions & Prospective payment & Continuity of care & \\
\hline Audit and feedback & Provider incentives & Arrangements for follow-up & \\
\hline (Computerized) reminders & Institution incentives & Case management (including co- & \\
\hline Marketing & Provider grant/allowance & ordination of assessment, treatment & \\
\hline \multirow[t]{26}{*}{ Mass media } & Institution grant/allowance & and arrangements for referrals). & \\
\hline & Provider penalty & Satisfaction of providers with the con- & \\
\hline & Institution penalty & ditions of work and the material and & \\
\hline & Formulary & psychological rewards & \\
\hline & Patient interventions & Communication and case discussion & \\
\hline & Premium & between distant health professionals & \\
\hline & Co-payment & Patient oriented interventions & \\
\hline & User-free & Mail order pharmacies & \\
\hline & Patient incentives & Presence and functioning of adequate & \\
\hline & Patient grant/allowance & mechanisms for dealing with patients' & \\
\hline & Patient penalty & suggestions and complaints. & \\
\hline & & Consumer participation in governance & \\
\hline & & of health care organizations & \\
\hline & & Structural interventions & \\
\hline & & Changes to the setting/site of service & \\
\hline & & delivery & \\
\hline & & Changes in physical structure, facilities & \\
\hline & & and equipment & \\
\hline & & Changes in medical record systems & \\
\hline & & Changes in scope and nature of ben- & \\
\hline & & efits and services. & \\
\hline & & Presence and organization of quality & \\
\hline & & monitoring mechanisms. & \\
\hline & & Ownership, accreditation, and affiliation & \\
\hline & & status of hospitals and other facilities. & \\
\hline & & Staff organization & \\
\hline
\end{tabular}

Notes: ${ }^{2} \mathrm{O}$ ther categories to be agreed in consultation with the EPOC editorial team.

\section{Participants and setting}

Nearly all participants in the included studies were medical doctors, either general practitioners or internal medicine residents, while 3 studies involved practice nurses or practice assistants in the implementation strategy. The location of care included hospital outpatient clinics in the UK and the USA (Hall et al 2003; Jacobson et al 2006) and GP surgeries in Norway, the UK, the Netherlands, and New Zealand (Hetlevik et al 1999; Peters et al 1999; Montgomery et al 2000; Fretheim et al 2006b; Sinclair and Kerr 2006; Van Steenkiste et al 2007).

\section{Characteristics of the studies}

The trials differed considerably in size: the smallest trial included 323 patients, while a bigger trial included 2239 patients. In this large study, however, the risk table was only applied to $12 \%(\mathrm{~N}=104)$ of the eligible patients
(Hetlevik et al 2000). In the majority of the studies, the proportion of patients who completed the proposed study protocol was small. One trial included no patients, but 343 GPs were asked to evaluate the intervention strategy (De Muylder et al 2005). This trial, as well as 5 other studies, may have suffered from a unit of allocation problem, particularly, a difference between the unit of allocation and the unit of analysis, without controlling for this by means of clustered data analysis.

Seven randomized controlled trials (RCT) were found, one of them a prospective RCT without follow-up (Hall et al 2003), the others varying in time until follow-up assessment from 6 to 18 months (Hetlevik et al 1999; Montgomery et al 2000; Jacobson et al 2006; Fretheim et al 2006b). One study reported on blinded assessment of the primary outcomes (Fretheim et al 2006b), while 5 studies mentioned concealment of intervention allocation. Primary outcomes 
Table 2 Medline search terms and strategy

\begin{tabular}{|c|c|c|}
\hline \multicolumn{3}{|l|}{ Basic search strategy (Brindle) } \\
\hline \#I chd risk assessment\$ & \#37 risk calculation\$ & \#72 (busselton adj2 score $\$$ ) \\
\hline$\# 2$ cvd risk assessment $\$$ & \#38 risk calculator $\$$ & $\# 73$ erica risk score $\$$ \\
\hline$\# 3$ heart disease risk assessment $\$$ & $\# 39$ risk factor $\$$ calculator $\$$ & \#74 framingham scor $\$$ \\
\hline$\# 4$ coronary disease risk assessment $\$$ & $\# 40$ risk factor $\$$ calculation $\$$ & \#75 dundee scor\$ \\
\hline$\# 5$ cardiovascular disease risk assessment\$ & $\# 4$ I risk engine $\$$ & \#76 brhs scor\$ \\
\hline \#6 cardiovascular risk assessment $\$$ & $\# 42$ risk equation $\$$ & \#77 British Regional Heart study risk scor\$ \\
\hline$\# 7 \mathrm{cv}$ risk assessment $\$$ & $\# 43$ risk table\$ & \#78 brhs risk scor $\$$ \\
\hline \#8 cardiovascular disease $\$$ risk assessment $\$$ & \#44 risk threshold\$ & \#79 dundee risk scor\$ \\
\hline \#9 coronary risk assessment $\$$ & \#45 risk disc? & \#80 framingham guideline $\$$ \\
\hline \#10 coronary risk scor $\$$ & \#46 risk disk? & \#8I framingham risk? \\
\hline \#II heart disease risk scor $\$$ & \#47 risk scoring method? & \#82 new zealand table $\$$ \\
\hline$\# 12$ chd risk scor $\$$ & \#48 scoring scheme? & \#83 ncep guideline? \\
\hline \#I3 cardiovascular risk scor $\$$ & \#49 risk scoring system? & \#84 smac guideline? \\
\hline \#I4 cardiovascular disease $\$$ risk scor $\$$ & $\# 50$ risk prediction? & \#85 copenhagen risk? \\
\hline \#I5 cvd risk scor\$ & \#5I predictive instrument? & \#86 or/57-85 \\
\hline$\# 16 \mathrm{cv}$ risk scor $\$$ & \#52 project\$ risk? & \#87 56 or 86 \\
\hline \#17 or/1-16 & \#53 cdss & \#88 exp decision support techniques/ \\
\hline \#18 cardiovascular diseases/ & \#54 or/28-53 & \#89 Diagnosis, Computer-Assisted/ \\
\hline \#19 coronary disease/ & \#55 27 and 54 & \#90 Decision Support Systems, Clinical/ \\
\hline \#20 cardiovascular disease $\$$ & \#56 17 or 55 & \#9I algorithms/ \\
\hline$\# 2$ I heart disease $\$$ & \#57 new zealand chart\$ & \#92 algorithm? \\
\hline$\# 22$ coronary disease $\$$ & \#58 sheffield table $\$$ & \#93 algorythm? \\
\hline \#23 cardiovascular risk? & \#59 procam & \#94 decision support? \\
\hline \#24 coronary risk? & \#60 General Rule to Enable Atheroma Treatment & \#95 predictive model? \\
\hline \#25 exp hypertension/ & \#6I dundee guideline $\$$ & \#96 treatment decision? \\
\hline \#26 exp hyperlipidemia/ & \#62 shaper scor $\$$ & \#97 scoring method $\$$ \\
\hline \#27 or/18-26 & \#63 (brhs adj3 score $\$$ ) & \#98 (prediction\$ adj3 method\$) \\
\hline \#28 risk function & \#64 (brhs adj3 risk\$) & \#99 or/88-98 \\
\hline \#29 Risk Assessment/mt (Methods) & \#65 copenhagen risk & \#100 Risk Factors/ \\
\hline$\# 30$ risk functions & \#66 precard & \#I0I exp Risk Assessment/ \\
\hline$\# 31$ risk equation $\$$ & \#67 (framingham adjl (function or functions)) & \#102 (risk? adjl assess $\$$ ) \\
\hline \#32 risk chart? & \#68 (framingham adj2 risk) & \#103 risk factor? \\
\hline \#33 (risk adj3 tool\$) & \#69 framingham equation & \#104 or/100-103 \\
\hline \#34 risk assessment function? & \#70 framingham model\$ & \#105 27 and 99 and 104 \\
\hline \#35 risk assessor & \#7I (busselton adj2 risk\$) & \#I0687 or $105(448.950)$ \\
\hline \multicolumn{3}{|l|}{ \#36 risk appraisal\$ } \\
\hline \multicolumn{3}{|l|}{ Adjustments for the current review } \\
\hline \#I07 106 and quality* health care & \#II6 II5 and clin* trial & \#1 29 I 28 and cardio* risk* \\
\hline \#108 106 and practice pattern* health care & \#II7 II5 and rand* trial & \#130 I 28 and risk tables \\
\hline \#109 106 and implement* strateg* & \#II8 II5 and randomised trial & \#13। I 28 and cardio* risk? \\
\hline \#II0 106 and implement* & $\# I 19$ II5 and randomized trial & \#132 I 28 and risk manag* \\
\hline \#III 106 and dissemination & $\# 120$ II5 and clinical trial & \\
\hline \#II2 106 and diffus* & $\#|2| I 15$ and cohort & $\# 133$ II 5 and process assessment \\
\hline \#II3 106 and guideline* & $\#|2|$ II5 and control* trial & \#134 II5 and process evaluation \\
\hline \#II4 I06 and implementation? & \#I22 II5 and comparat* study & $\# 135$ II5 and \#I 7 \\
\hline \multirow[t]{6}{*}{ \#II5 107-II4 (18607) } & $\# 123$ II5 and random* control* trial & \\
\hline & $\# 124$ II5 and observat* study & \\
\hline & $\# 125$ II5 and rand* clin* trial & \\
\hline & $\# 126$ II5 and control* study & \\
\hline & \#127 II5 and rand* study & \\
\hline & \#128 I I6-127 & \\
\hline
\end{tabular}




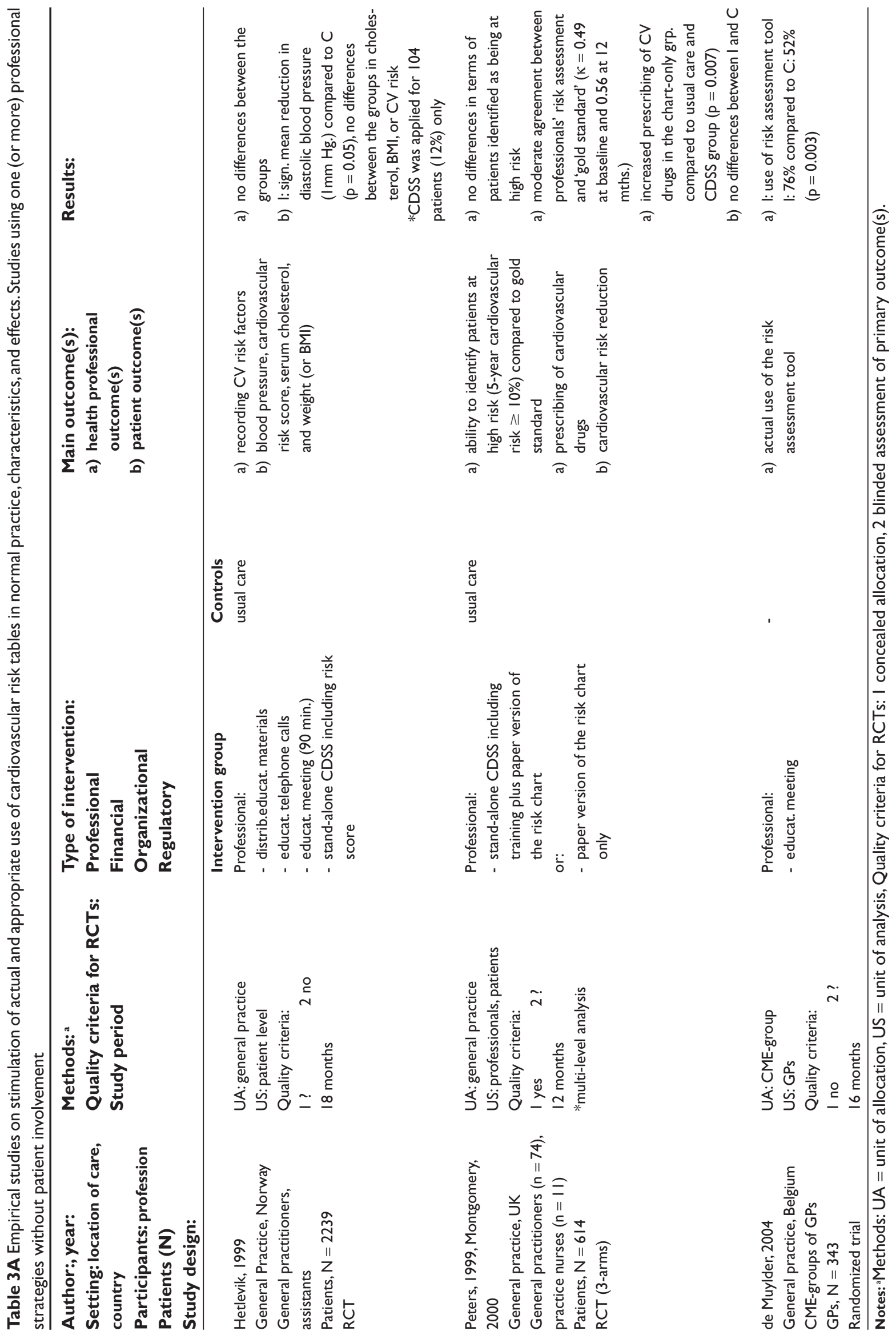




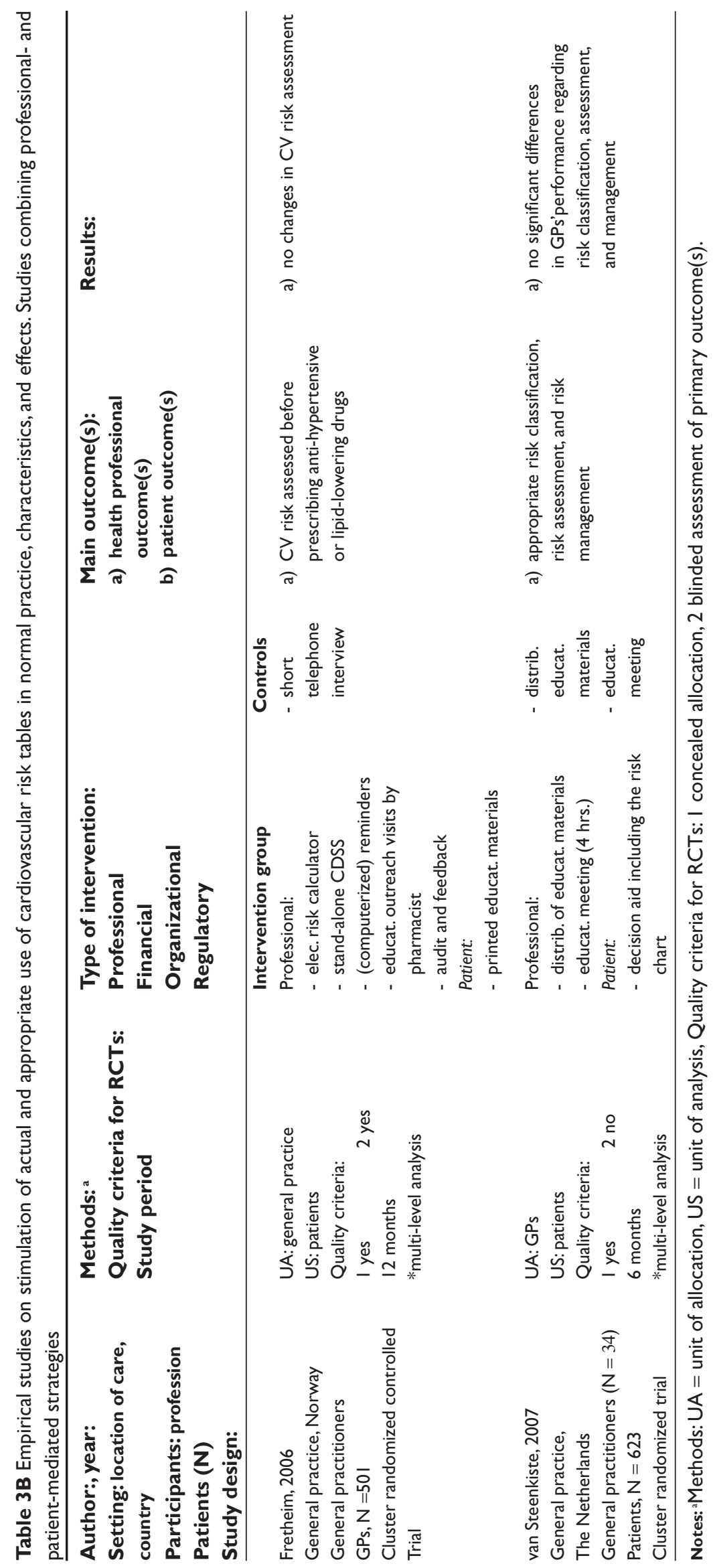




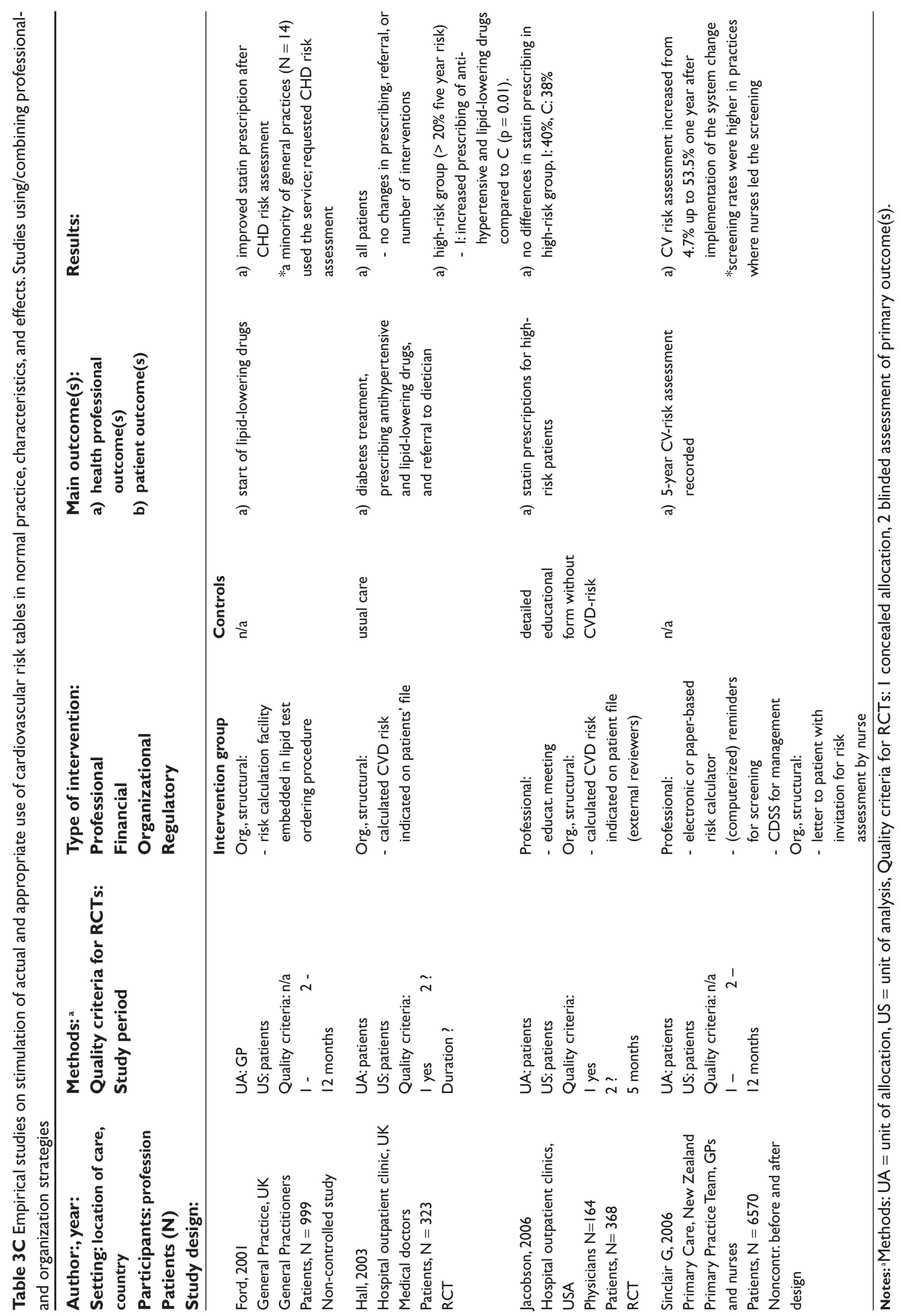


relating to the health professionals were the recording of CV risk and risk factors, the ability to identify patients at high risk, and appropriate indications for treatment (prescribing of cardiovascular drugs). Secondary, patient-related outcomes concerned $\mathrm{CV}$ risk and risk reduction, blood pressure, serum cholesterol, body mass index, and self-reported lifestyle.

\section{Type of intervention and effects}

We found that the interventions in the included studies were basically limited to three types: professional strategies or combinations of them (without patient involvement); combinations of professional- and patient-mediated strategies; organizational strategies or combinations of professional and organizational strategies. In general, the effects of the interventions on primary outcomes were small, absent or contradictory for the same outcome.

\section{Professional strategies}

Education was the single intervention in one of the three 'professional-oriented' trials. This was found to significantly increase the use of the risk tables (De Muylder et al 2005). The other two studies involved one or more educational meetings for transfer of knowledge, in combination with training in the use of a stand-alone computerized decision support system (CDSS) (Hetlevik et al 1999; Montgomery et al 2000). In the 3-armed trial by Montgomery, the use of the CDSS plus a paper version of the risk tables, or a paper version only versus usual care, had no effect on the ability to identify patients at high cardiovascular risk or on more accurate use of the risk tables. Although increased prescribing of CV drugs and a significantly lower systolic blood pressure was seen in the 'risk chart only' group, no differences in risk reduction were found compared to usual care (Peters et al 1999; Montgomery et al 2000). Interventions in which the educational meetings and the use of a CDSS were supplemented with educational materials, follow-up telephone calls and feedback on actual performance had no effect on the recording of risk factors, cholesterol level, BMI, or CV risk. However, a significant mean reduction in diastolic blood pressure (1 mm HG) was reported (Hetlevik et al 1999).

\section{Professional- and patient-mediated strategies}

Two trials combined professional interventions, such as education plus audit and feedback, with a patient-mediated intervention, involving new information on patients' CV risk being made available at the consultation (Fretheim et al 2006b), and introducing a decision aid for patients (Van Steenkiste et al 2007). The trial by Fretheim and colleagues (2006b) used an outreach visit to educate GPs on $\mathrm{CV}$ prevention and risk communication, as well as to give feedback on actual performance, and train them to use a CDSS. The CDSS generated 'pop-ups' on screen whenever an elevated blood pressure or cholesterol level was recorded, had a CV-risk calculator, generated treatment advice, and could be used to print patient-education materials. It had no effect on CV-risk assessment prior to prescribing (Fretheim et al 2006b). The trial by Van Steenkiste and colleagues (2007) used a combination of a 4-hour training session in the use of the risk tables, risk communication, and a decision aid for patients, which was to be used in two consultations to ensure patient involvement in the second consultation. Neither resulted in improved performance of the GPs in terms of risk classification, assessment, or management (Van Steenkiste et al 2007).

\section{Organizational}

The focus of the interventions in this group of 4 studies (2 of which were noncontrolled) was a change in facilities and equipment. In one of the two controlled trials, patients' CV risk was clearly indicated (by the researcher) on the front page of the patients' file. This had no effect on prescribing behavior, treatment or referral, except that a significant increase in the use of CV drugs was observed for the high-risk group (Hall et al 2003). The other controlled trial involved adding an educational meeting of the professionals, and contrasted a low-detail educational form including CV risk on the patients' file in the intervention group with a more detailed form without $\mathrm{CV}$ risk in the control group. This resulted in overuse of statins in the moderate-risk group and no effect in the high-risk group (Jacobson et al 2006).

One of the two noncontrolled studies evaluated a risk-calculating facility embedded in a lipid test ordering procedure. Statin prescribing was found to be improved after risk assessment, but only 14 GPs used the laboratory service involved in the study (Ford et al 2001). The other study used a CDSS with risk calculator, integrated in the practice management system, which linked pop-up alerts to pre-selected files that appeared whenever the patient file was opened. In addition, the eligible patients were sent a letter explaining the value of screening for $\mathrm{CV}$ risk and inviting them for a risk assessment. CV-risk assessment had increased from $4.7 \%$ to $53.5 \%$ one year after implementation of the system change. Screening rates were higher in practices where nurses were responsible for the screening. 


\section{Discussion}

The small number of studies that could be included in this systematic review shows that the development of effective strategies for the implementation of cardiovascular risk tables in primary prevention has been sparsely studied so far, and that our understanding of ways to speed up the uptake of such new tools remains limited. The most common type of strategy was organizational, involving methods like presenting CV risk on the front page of patients' files or as a laboratory facility, and pro-active screening by pop-up alerts on the computer screen with invitations sent to patients' homes for risk assessment. Patient-mediated implementation strategies have hardly been studied. Although no clear relation between a particular type of strategy and the success or failure of implementation was observed, promising strategies seemed to be those involving teamwork, nurse-led clinics and integrated IT support.

This review used a sensitive search, with language restricted to Dutch, English, and German publications. Although a restriction to randomized controlled trials is often desirable in systematic literature reviews, as it yields studies with the highest level of evidence, we chose to include non-controlled and quasi-controlled intervention studies as well, because of the expected low number.

Most of the studies we found do not seem to be theorydriven or problem-driven (ie, tailored to barriers and facilitators), although in two studies, the strategy was guided by a systematic survey of barriers and facilitators before implementation was started (Fretheim et al 2006b; Van Steenkiste et al 2007). Despite this, no effects were found in these studies. Apart from a qualitative process evaluation, there is a need for more in-depth qualitative methods, eg, indepth interviews or focus groups with health professionals, to increase our understanding of this lack of effect (Fretheim et al 2006a). Ideally, this should be done along the lines of established planning models for implementation processes. It seems that insufficient attention has been given to the various phases in the process of change for care providers and teams (Grol and Grimshaw 2003; Grol and Wensing 2004).

Although the theories and models on organizational determinants of innovation of care processes overlap, and the empirical evidence behind their assumptions about human behavior or organizational change is limited, we tried to apply our findings to some of the many available theories. The Theory on Innovative Organizations tells us to take the type of organization into account. Decentralized decision making about innovations is crucial and good teamwork is one of the main keys for successful change. Process Reengineering
Theory advises to focus on multidisciplinary care processes and collaboration instead of individual decision making. The theory aims to analyze and redesign the work process related to CVD prevention. The lessons learnt from this review, such as task delegation to a practice nurse and sending invitational letters to patients for screening, could be part of it. Theories on Organizational Culture stress that changes in the culture can stimulate changes in performance; more teamwork, flexibility and external orientation. Organizational Learning Theory advises to create conditions within the (practice) organization for continuous learning at all levels (Grol et al 2007).

Although financial incentives are an effective way of changing professional behavior, none of the studies we found used a financial incentive for the implementation of risk tables. It might be a powerful strategy, since most professionals might in the near future encounter financial incentives that are linked to the quality of care, as is already customary in the UK. Many indicators of the Quality and Outcomes Framework are related to prevention of CVD. It appears that significant health gains could result from achieving these quality targets (McElduff et al 2004; Roland 2004; Sutton and McLean 2006; Campbell et al 2007).

Ideally, implementation strategies should be tailored to support physicians' needs, and as such should be based on the problems they are actually facing (Grol and Grimshaw 2003; Grol and Wensing 2004). We distinguished 5 needs related to CVD prevention, and propose recommendations for implementation based on the review:

1. Supporting physicians in the process of case-finding of potential high-risk patients. This is an essential step in successful application of the risk tables. Active support can be provided by pop-up alerts linked to pre-selected files that appear whenever a patient file is opened. Letters sent to patients' homes, explaining the value of screening for CV risk and with an invitation for risk assessment, can further improve screening rates, especially in those practices with an established culture of practice-nurse led clinics and teamwork between the practice nurses and the GPs.

2. Supporting physicians in completing the risk profile. A CDSS proved to be effective only when the decision support system was integrated in the practice management system.

3. Supporting physicians in calculating the risk. Most of the studies promoted the use of a risk table, either paper-based or computerized, for risk calculation. The main problem seemed to be the actual use of the risk 
calculation tools, which many of the physicians failed to do. Initially, it was suggested that risk tables would be useful for clinicians to help them master concepts relating to $\mathrm{CHD}$ prevention, ie, as a kind of educational tool rather than a practice instrument for risk calculation and management (Jackson and Beaglehole 1995). Since nurses are likely to be the future consumers (Will 2005), GPs could be supported by nurses in this task. Presenting the actual risk to the GPs, eg, by indicating the risk on the front page of patients' files, only had a minor effect on physicians' behavior in terms of prescribing $\mathrm{CV}$ drugs. Nevertheless, confronting GPs with their patients' actual risk may have corrected their misperceptions and could as such help to generate a better performance, as misperception of cardiovascular risk is not uncommon among health professionals (Peters et al 1999; De Muylder et al 2004; Frijling et al 2004; Mosca et al 2005; Graham et al 2006; Van der Weijden et al 2007).

4. Supporting physicians in risk communication. Once the risk has been calculated the next challenge is how to present it in an effective way. Most patients have a hard time understanding CVD risks (Erhardt and Hobbs 2002; Van Steenkiste et al 2004). Perception of risks tends to be inaccurate and people find it difficult to handle risks (Elwyn et al 2001). The format (framing) in which risk information is presented affects people's perception of risks and decision-making (Timmermans et al 2004). For example, framing in relative risk or loss framing are more persuasive compared to framing in absolute risk or gain framing respectively. This is reported for doctors (Rakow 2001), and patients (Edwards et al 2001; Feldman Stewart et al 2000; Lipkus et al 2001). Risk communication should include weighing up of risks and benefits of a treatment choice, and address the patient's perception of probability of an event as well as the value of the event for that individual (Edwards et al 2001). To achieve this, health professionals need training to increase their competences in risk communication.

5. Supporting physicians in deciding, jointly with the patient, on appropriate action for management. The use of a computerized decision support system to help physicians decide on appropriate management may result in some small favorable effects on prescribing behavior and blood pressure. So far, involving patients in the decision on appropriate management with the help of educational materials (including the risk table) has had no effect. Although patient involvement seems to be an effective prevention strategy, it is still unclear how this can be achieved (Edwards and Elwyn 1999; Edwards et al 2003). A successful strategy might be to delegate this task to a nurse or health coach, who has more time to explain CV risk and for patient involvement in decisions on management and follow-up (Vale et al 2003; Sol et al 2005; Edelman et al 2006). Such a strategy is a subject of research in the current IMPALA trial (ISRCTN51556722), in which a risk communication tool and a decision aid are issued to patients for preparation at home, and in which the practice nurse applies an adapted motivational interviewing technique to discuss the risk and options for risk reduction (see www.trialregister.nl).

\section{Conclusion}

Effective primary prevention of cardiovascular diseases is not easy, and research on effective strategies for the implementation of risk tables has been sparse. Nevertheless, good teamwork, nurse-led clinics, and smart software programs, integrated in the practice management system, seem to be promising strategies for patient selection and risk assessment. Achieving patient involvement, a precondition for successful CV-risk management, is a challenge for future research that, together with the development of other professional- and organization-implementation strategies, needs to be embedded in the methodology of implementation science.

\section{Disclosure}

Grant support: By the Netherlands Organization for Health Research and Development (ZonMw).

\section{References}

Brindle P, Beswick A, Fahey T, et al. 2006. The accuracy and impact of risk assessment in the primary prevention of cardiovascular disease: A systematic review. Heart, 92:1752-59.

Campbell S, Reeves D, Kontopantelis E, et al. 2007. Quality of primary care in England with the introduction of pay for performance. $N$ Engl J Med, 357:181-90.

Conroy RM, Pyorala K, Fitzgerald AP, et al. 2003. Estimation of ten-year risk of fatal cardiovascular disease in Europe: the SCORE project. Eur Heart J, 24:987-1003.

De Koning JS, Klazinga NS, Koudstaal PJ, et al. 2004. Quality of care in stroke prevention: results of an audit study among general practitioners. Prev Med, 38:129-36.

De Muylder R, Lorant V, Paulus D, et al. 2004. Obstacles to cardiovascular prevention in general practice. Acta Cardiol, 59:119-25.

De Muylder R, Tonglet R, Nackers F, et al. 2005. Randomised evaluation of a specific training of general practitioners in cardiovascular prevention. Acta Cardiol, 60:199-205.

Eaton CB, Galliher JM, McBride PE, et al. 2006. Family physician's knowledge, beliefs, and self-reported practice patterns regarding hyperlipidemia: a National Research Network (NRN) survey. $J$ Am Board Fam Med, 19:46-53. 
Edelman D, Oddone EZ, Liebowitz RS, et al. 2006. A multidimensional integrative medicine intervention to improve cardiovascular risk. $J$ Gen Intern Med, 21:728-34.

Edwards A, Elwyn G. 1999. The potential benefits of decision aids in clinical medicine. JAMA, 282:779-80.

Edwards A, Elwyn G, Covey J, et al. 2001. Presenting risk information--a review of the effects of "framing" and other manipulations on patient outcomes. $J$ Health Comm, 6:61-82.

Edwards A, Unigwe S, Elwyn G, et al. 2003. Personalised risk communication for informed decision making about entering screening programs. Cochrane Database Syst Rev, 1:CD001865.

Elwyn G, Edwards A, Eccles M, et al. 2001. Decision analysis in patient care. Lancet, 358:571-4.

Erhardt L, Hobbs FD. 2002. Public perceptions of cardiovascular risk in five European countries: the react survey. Int J Clin Pract, 56:638-44.

Feldman Stewart D, Kocovski N, McConnell BA, et al. 2000. Perception of quantitative information for treatment decisions. Med Decis Mak, 20:228-38.

Ford Dr, Walker J, Game FL, et al. 2001 Effect of computerized coronary heart disease risk assessment on the use of lipid-lowering therapy in general practice patients. Coronary Health Care, 5:4-8.

Fretheim A, Havelsrud K, Oxman AD. 2006a. Rational Prescribing in Primary care (RaPP): process evaluation of an intervention to improve prescribing of antihypertensive and cholesterol-lowering drugs. Implement Sci, 1:1-19.

Fretheim A, Oxman AD, Havelsrud K, et al. 2006b. Rational prescribing in primary care (RaPP): a cluster randomized trial of a tailored intervention. PLoS Med, 3:e134.

Frijling BD, Lobo CM, Keus IM, et al. 2004. Perceptions of cardiovascular risk among patients with hypertension or diabetes. Patient Educ Couns, 52:47-53.

Graham IM, Stewart M, Hertog MG. 2006 Factors impeding the implementation of cardiovascular prevention guidelines: findings from a survey conducted by the European Society of Cardiology. Eur J Cardiovasc Prev Rehabil, 13:839-45.

Grimshaw J, McAuley LM, Bero LA, et al. 2003. Systematic reviews of the effectiveness of quality improvement strategies and programmes. Qual Saf Health Care, 12:298-303.

Grol R. 1997. Beliefs and evidence in changing clinical practice. BMJ, 315:418-21.

Grol R. 2001. Improving the quality of medical care: building bridges among professional pride, payer profit, and patient satisfaction. JAMA, 286:2578-85.

Grol R. Grimshaw J. 2003. From best evidence to best practice: effective implementation of change in patients' care. Lancet, 362:1225-30.

Grol R. Wensing M. 2004. What drives change? Barriers to and incentives for achieving evidence-based practice. Med J Aust, 180:57-60.

Grol RP, Bosch MC, Hulscher ME, et al. 2007 Planning and studying improvement in patient care: the use of theoretical perspectives Milbank Q, 85:93-138.

Hall LM, Jung RT, Leese GP. 2003. Controlled trial of effect of documented cardiovascular risk scores on prescribing. BMJ, 326:251-2.

Hetlevik I, Holmen J, Kruger O. 1999. Implementing clinical guidelines in the treatment of hypertension in general practice. Evaluation of patient outcome related to implementation of a computer-based clinical decision support system. Scand J Prim Health Care, 17:35-40.

Hetlevik I, Holmen J, Kruger O, et al. 2000. Implementing clinical guidelines in the treatment of diabetes mellitus in general practice. Evaluation of effort, process, and patient outcome related to implementation of a computer-based decision support system. Int J Technol Assess Health Care, 16:210-27.

Jackson R, Beaglehole R. 1995. Evidence-based management of dyslipidaemia. Lancet, 346:1440-2.

Jacobson TA, Gutkin SW, Harper CR. 2006. Effects of a global risk educational tool on primary coronary prevention: the Atherosclerosis Assessment Via Total Risk (AVIATOR) study. Curr Med Res Opin, 22:1065-73.
Lipkus IM, Klein WM, Rimer BK. 2001. Communicating breast cancer risks to women using different formats. Cancer Epidemiol Biomarkers Prev, 10:895-8.

McElduff P, Lyratzopoulos G, Edwards R, et al. 2004. Will changes in primary care improve health outcomes? Modelling the impact of financial incentives introduced to improve quality of care in the UK. Qual Saf Health Care, 13:191-7.

Montgomery AA, Fahey T, Peters TJ, et al. 2000. Evaluation of computer based clinical decision support system and risk chart for management of hypertension in primary care: randomised controlled trial. $B M J$, 320:686-90.

Mosca L, Linfante AH, Benjamin EJ, et al. 2005. National study of physician awareness and adherence to cardiovascular disease prevention guidelines. Circulation, 111:499-510.

Peters TJ, Montgomery AA, Fahey T. 1999. How accurately do primary health care professionals use cardiovascular risk tables in the management of hypertension? Br J Gen Pract, 49:987-8.

Rakow T. 2001. Differences in belief about likely outcomes account for differences in doctors' treatment preferences: but what accounts for the differences in belief? Qual Health Care, 10(Suppl 1)i44-9.

Roland M. 2004. Linking physicians' pay to the quality of care - a major experiment in the United kingdom. $N$ Engl J Med, 351:1448-54.

Shaw B, Cheater F, Baker R, et al. 2005. Tailored interventions to overcome identified barriers to change: effects on professional practice and health care outcomes. Cochrane Database Syst Rev, 1:CD005470.

Sheridan S, Pignone M, Mulrow C. 2003. Framingham-based tools to calculate the global risk of coronary heart disease: a systematic review of tools for clinicians. J Gen Intern Med, 18:1039-52.

Sinclair G, Kerr A. 2006. The Bold Promise Project: a system change in primary care to support cardiovascular risk screening. $N Z$ Med $J$, 119:U2312.

Sol BG, Van Der Bij1 JJ, Banga JD, et al. 2005. Vascular risk management through nurse-led self-management programs. $J$ Vasc Nurs, 23:20-4.

Sutton M, McLean G. 2006. Determinants of primary medical care quality measured under the new UK contract: cross sectional study. $B M J$, 332:389-90.

Thomas S, Van Der Weijden T, Van Drenth BB, et al. 1999. NHG-Standaard Cholesterol (eerste herziening). Huisarts Wet, 42:406-17.

Timmermans D, Molewijk B, Stiggelbout A, et al. 2004. Different formats for communicating surgical risks to patients and the effect on choice of treatment. Patient Educ Couns, 54:255-63.

Torley D, Zwar N, Comino EJ, et al. 2005. GPs' views of absolute cardiovascular risk and its role in primary prevention. Aust Fam Physician, 34:503-4, 7 .

Vale MJ, Jelinek MV, Best JD, et al. 2003. Coaching patients On Achieving Cardiovascular Health $(\mathrm{COACH})$ : a multicenter randomized trial in patients with coronary heart disease. Arch Intern Med, 163:2775-83.

Van Der Weijden T, Steenkiste Van B, Stoffers HE, et al. 2007. Primary prevention of cardiovascular diseases in general practice; mismatch between cardiovascular risk and patients' risk perceptions. Med Decis Mak, 6:754-61.

Van Steenkiste B, Van Der Weijden T, Timmermans D, et al. 2004. Patients' ideas, fears and expectations of their coronary risk: barriers for primary prevention. Patient Educ Couns, 55:301-7.

Van Steenkiste B, Van Der Weijden T, Stoffers HE, et al. 2007. Improving cardiovascular risk management: a randomized, controlled trial on the effect of a decision support tool for patients and physicians. Eur $J$ Cardiovasc Prev Rehabil, 14:44-50.

Wang TJ, Gona P, Larson MG, et al. 2006. Multiple biomarkers for the prediction of first major cardiovascular events and death. $N$ Engl $J$ Med, 355:2631-9.

Will CM. 2005. Arguing about the evidence: readers, writers and inscription devices in coronary heart disease risk assessment. Sociol Health Ill, 27:780-801. 
